\title{
FORMACIÓN DE GESTORES COMUNICACIONALES DE SALUD COMUNITARIA MEDIANTE INTEGRACIÓN UNIVERSITARIA
}

\author{
TRAINING OF COMMUNICATION MANAGERS OF COMMUNITY HEALTH THROGH \\ UNIVERSITY INTEGRATION
}

\section{RESUMEN}

El artículo plantea una síntesis de reflexión basada en la observación, la experiencia alcanzada en el programa de formación de especialistas en Medicina Familiar y en estudios bibliográficos, en tres contextos académicos: la Universidad del Llano Ezequiel Zamora (UNELLEZ) en Venezuela, Universidad de Ciencias Médicas de La Habana, en la Facultad de Tecnología de la Salud (FATESA) en Cuba y en la Escuela Superior Politécnica de Chimborazo (ESPOCH) en Ecuador. El objetivo del artículo es fundamentar la necesidad de la formación por competencias, de profesionales integrales de salud, mediante la integración universitaria, para mejorar la calidad de vida comunitaria. El estudio realizado, se considera pertinente, como insumo para la respuesta a la problemática social planteada, para dotar de competencias comunicacionales tanto a educandos de las ciencias salubristas, que poseen debilidades pedagógicas, como a los profesionales de formación pedagógica que no cuentan con conocimientos en áreas de la salud. El impacto de la investigación se reflejará en la justificación de la formación de profesionales dotados de integralidad con sólidas competencias comunicativas, con ética y valores, que incida en el contexto comunitario.

Palabras clave: Gestores comunicacionales de salud, prevención y promoción, formación por competencias, integración universitaria.

\section{ABSTRACT}

The article presents a synthesis of reflection based on observation, experience and bibliographic studies, this has been developed in three academic contexts: University of Llano Ezequiel Zamora (UNELLEZ) in Venezuela, University of Medical Sciences of Havana, Faculty of Technology of Health (FATESA) in Cuba and the Polytechnic School of Chimborazo (ESPOCH) in Ecuador in the training program for specialists in Family Medicine. The objective of this article is to support the need for training by competencies of integral health professionals. This can be achieved through university integration to improve the quality of communitarian living style. The study was relevant, as an input for the response to a social problem to provide communicative competencies to students of health sciences, who have pedagogical weaknesses, as well as to the pedagogical training professionals who do not have knowledge in the areas of health. The impact of the research will be reflected in the justification of the training of professionals endowed with integrality with solid communicative competences, with ethics and values, which affects the community context.

Keywords: communicational managers of health, prevention and promotion, competency-based training, university integration.

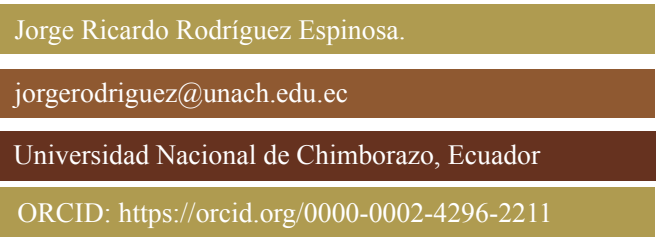




\section{INTRODUCCIÓN}

La sociedad postmoderna exige una acción científica con formación axiológica elevada, es decir una ética que responda a los principios que rigen la investigación de estos tiempos, además requiere de un cambio y fortalecimiento en la comunicación en salud, pues la salud es un derecho de todos y un deber obligatorio del Estado.

En las últimas décadas, se ha avanzado en algunos de los indicadores globales de salud en la región, alcanzándose, por ejemplo, una tasa de mortalidad infantil de 27,7 por 1.000 y una expectativa de vida al nacer cercana a los 72 años para el quinquenio 2000-2005. La transición demográfica y epidemiológica se expresa en un incremento progresivo de la población de 60 años y más que, según estimaciones, aumentaría desde un $8 \%$ en el año 2000 , a un $14,1 \%$ el año 2025 $\mathrm{y}$, a la vez, en un peso relativamente creciente de condiciones como el cáncer, las enfermedades crónicas, los trastornos de salud mental, el VIH/SIDA y los accidentes en el perfil de salud. (Adler et al. 1994).

La experiencia cubana en el ámbito de salud se ha llevado a varios países a través de la Alternativa Bolivariana para las Américas (ALBA), la cual alcanza niveles cimeros internacionalmente, con la conjunta formación del médico especialista en Medicina Familiar y Comunitaria.

Cuba cuenta con profesionales Máster en Educación para la Salud, sin embargo, no se ha logrado consolidar competencias comunicacionales que integren las áreas de Pedagogía y la de Salud. Los congresos de ambas ciencias, han servido de plataforma para la socialización discursiva, pero el impacto en la comunidad aún no es el ideal. Una de las posibles causas es la verticalización de estos especialistas en enfermedades determinadas, descuidando los procesos de comunicación globales que exige el sistema de salud de manera integral.

En el campo de la salud, la actividad fundamental de los profesionales que trabaja- ban en la asistencia ambulatoria del nivel primario, estaba básicamente orientada a la atención curativa o rehabilitadora de las personas enfermas que acudían a las consultas en demanda de solución a sus problemas de salud.

El concepto de promoción más actualizado, en salud pública, data de la década de los 70 , enunciado por Lalonde en el 1974, aunque sus bases históricas son muy remotas según refiere Atria en el 2003. La promoción de salud se hace muy útil cuando se inicia en los años 70- 80, los tres niveles de atención en salud, surgiendo su importancia en el nivel primario de salud.

"La promoción de la salud es el proceso que permite a las personas incrementar el control sobre su salud para mejorarla" (Martínez 2009). La Organización Mundial de la Salud (OMS) define como promoción de salud, el proceso que les permite a todas las personas cuidar del mantenimiento de su salud para mejorar el estilo y la calidad de vida.

Se trata de un proceso político y social no sólo dirigido a fortalecer las capacidades y habilidades, sino también a lograr un mayor control de los determinantes de salud y modificar las condiciones sociales, económicas y ambientales de los individuos en favor de la salud pública e individual (OMS 1986:10).

La visión holística que plantea Bracht, en cuanto a la promoción de salud, implica la necesidad de abordar el desarrollo comunitario como esencial, esto hace que la acción de cooperación que tiene la comunidad se favorezca. Esto hace que se asegure el acceso distributivo a la educación, la seguridad de la economía y el apoyo social sostenible, de acuerdo al contexto adecuado de las políticas públicas de acuerdo a los objetivos trazados por los gobiernos de cada país. (Bracht 1999). El concepto de promoción de salud, en el sentido en que actualmente se le conoce, se hace presente en salud pública desde la década de los 70, aunque sus bases históricas son muy remotas y a partir de esa fecha ha sufrido cambios positivos, dentro de la salud pública, sobre todo de América Latina. El progreso de los nuevos conceptos que 
caracterizan a la moderna Atención Primaria de salud, está permitiendo, avanzar en la implementación de actividades preventivas de enfermedades y promoción de salud en el seno de la formación de equipos multidisciplinarios, estos sirven además para una adecuada atención de este nivel. Alcanzando una prioridad cada vez mayor, hasta convertirse en el elemento nuclear del cambio cualitativo en la transformación del sistema sanitario que se está llevando a cabo en muchos países en la actualidad, como España, Uruguay y en especial Cuba, que ha llevado esta experiencia a Venezuela, Bolivia, Ecuador, Brasil, entre otros.

Cuba ha realizado labores de misiones médicas en el extranjero, a través de educación para la salud en pregrado y postgrado como, por ejemplo, en 2003 en Venezuela, UNELLEZ de San Fernando de Apure, Barrio Adentro, se realizó una nivelación con todos los postulantes a ingresar en la carrera de Medicina Integral Comunitaria (MIC), para formarlos como promotores de salud y luego matricular en las aulas de medicina.

En el año 2012, en la ESPOCH, Ecuador, se trabajó en el proceso académico de posgrado, con la formación de especialistas en Medicina Familiar Comunitaria (MFC), donde se fortaleció el trabajo de educación para la salud en la comunidad.

En el desarrollo de dichas actividades se observó la inminente necesidad de fortalecer las competencias comunicativas para lograr un mayor impacto y mejores resultados en las comunidades donde el profesional interviene, de ahí es que parte el objetivo del artículo que es fundamentar la necesidad de la formación de profesionales integrales de salud, mediante la integración universitaria. En síntesis este trabajo, fundamenta la necesidad de la formación educacional del gestor comunicacional de salud comunitaria, inmersa en los tres escenarios fundamentales de los estudios universitarios; la formación académica, la investigación y la vinculación con la sociedad, con lo que se pudiera lograr que el futuro gestor de salud, cuente con los valores y experiencias, que le permitan desarrollar habilidades pedagógicas y habi- lidades cognitivas inherentes al campo de la promoción de salud, sobre todo en la atención primaria, con la pertinencia requerida, así como competencias socio formativas, para dar respuesta efectiva a los problemas en su contexto laboral.

De tal forma se potenciaría la constitución de un profesional integral, humanitario, altruista, solidario, con sólidas competencias comunicativas en la promoción de la salud, que cause un impacto social en el contexto comunitario, que sea capaz de difundir los conocimientos adquiridos y multiplicar sus efectos a través de líderes no formales.

\section{METODOLOGÍA}

Este trabajo es un artículo de reflexión que se origina en la investigación de tesis doctoral, efectuada por el autor, en el área de Ciencias Pedagógicas, en la Universidad "Enrique José Varona", de La Habana, Cuba, a partir del año 2015, donde utiliza las experiencias adquiridas como docente universitario de pregrado y posgrado, en la formación de profesionales de la salud.

También se toma en cuenta la experiencia como docente-investigador en la Facultad de Tecnología de la Salud de la Universidad de Ciencias Médicas de La Habana, Cuba, en el período 2003 a 2015 y el accionar como docente de postgrado de Medicina Familiar Comunitaria, en la ESPOCH de Ecuador. Estas tres experiencias permitieron, detectar debilidades en las competencias comunicativas en los profesionales salubristas, que fueron corroboradas en el análisis crítico de la obra de varios autores, para llegar a la reflexión de este artículo.

La metodología utilizada, se basa en las experiencias empíricas, la observación, el análisis sintético de los programas curriculares y del material bibliográfico revisado en los escenarios de acción descritos, utilizando los métodos: histórico-lógico, análisis y síntesis, inducción y deducción y la técnica de análisis documental, con énfasis en el desarrollo de la salud en Cuba, como modelo replicado en otros países de Latinoamérica y el mundo.

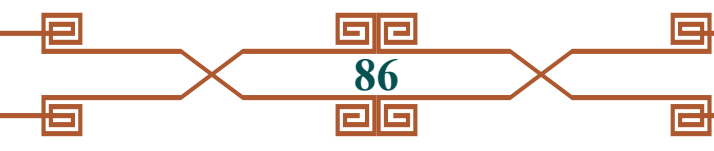




\section{RESULTADOS Y DISCUSIÓN}

\section{Rol de la Universidad en el proceso de formación.}

Por la importancia de la universidad en el proceso de formación, la Educación Superior ha sido declarada por la CRES 2008 (Conferencia Regional de Educación Superior) y por la CMES 2009 (Conferencia Mundial de Educación Superior) como un derecho y bien público social, según Alarcón, responsabilizando así a los Estados para garantizarla, en condiciones de equidad y calidad para un desarrollo educacional sostenible (Alarcón 2014).

La universidad actual, a nivel mundial, debe verse desde el plano innovador e integrador. Esto exige que se proporcione una equidad en la docencia, junto a la investigación y ambas vinculadas a la sociedad. Para que se produzca este fenómeno deben existir la voluntad política y los profesionales preparados para este proceder.

En el caso de Cuba, la universidad tiene su verdadero desarrollo a partir del año 1959 , como parte de todo el conjunto de profundas transformaciones sociales que hubo desde ese momento, basada en nuevos conceptos de equidad y justicia social, que ha ido conduciendo a alcanzar el reconocido prestigio en el campo educativo que tiene hoy.

Es una universidad científica, tecnológica y humanista la cual es caracterizada por estas tres cualidades. Su carácter científico expresado en su gradual contribución como centro de investigación científica, dado que, profesores y estudiantes se vinculan a esta tarea. El segundo rasgo es el desarrollo tecnológico que constituye un pilar fundamental con una amplia red de carreras de perfiles tecnológicos, que aseguran la introducción de nuevos avances en empresas, industrias, instalaciones productivas y de servicios.

Horruitiner, en su investigación, menciona el carácter humanístico de la universidad, lo cual es muy importante en la comprensión de una concepción universitaria con la base en la formación de lo instructivo, lo cognitivo y se centre la atención en el pleno desarrollo de la personalidad del hombre, consciente del compromiso social que tiene, de la ética, de los valores que devienen de la prioridad de estos principios (2005).

La educación superior forja la identidad nacional, contribuye al desarrollo de las generaciones venideras, a la cohesión social, a fortalecer y fomentar los valores de los ciudadanos, aportando a la construcción de las seguridades humanas y a la promoción de la cultura de paz; y para ello es fundamental implicar la pertinencia educacional y la sostenibilidad de la integralidad del sistema educativo.

Resulta de especial interés para la sociedad, que las universidades o instituciones de educación superior desarrollen investigaciones y difundan conocimientos que incorporen valor social y cultural a los procesos de ejecución y fortalecimiento de las áreas estratégicas de desarrollo, a través de una ciencia y tecnología innovadora, enmarcada en diferentes campos.

De forma tal que permitan el redimensionamiento de su papel en la construcción de lo público, entendido este como un espacio de participación ciudadana para el debate, el ejercicio de responsabilidades, a fomentar sus valores, así como para el conocimiento, la cimentación y profundización de los derechos humanos, ya estipulados, al servicio de los más necesitados y del mantenimiento de una sociedad más justa y equitativa.

En cuanto a la sociedad y la universidad, la intervención en los procesos sociales que llevan adelante la educación superior actual deberá convertirse en una oportunidad para reflexionar, consolidar y gestionar en los sistemas de gestión universitaria.

Así como sus principios, funciones, responsabilidades, lenguajes, paradigmas, la formación profesional y las concepciones de la calidad que promueva el desarrollo de los actores y sectores educativos, que genere procesos sistémicos y complejos de organización, que establezca metodologías para concebir impactos en el desarrollo social, plantee oportunidades para la producción, 
la gestión social y productiva- cultural del conocimiento, reconociendo la diversidad de contextos, historias y trayectorias institucionales.

\section{Fortalecimiento de las competencias co- municativas en la educación para la salud.}

Al hablar de la formación de personas con competencias se establecen responsabilidades a tomar en cuenta por cada uno de los integrantes de la sociedad. Detallándose así la responsabilidad social que exige vivir en convivencia, solidaridad y cooperación.

El gobierno debe estimar de manera continua la calidad de evaluación en la educación, a partir de ello implementar acciones concretas que aseguren que el servicio educativo esté acorde con las necesidades de las personas, los retos sociales, laborales, profesionales y culturales de las instituciones educativas, que deben brindar el servicio educativo acorde con los más altos estándares de calidad, en el marco de las políticas públicas nacionales e internacionales.

Las organizaciones sociales y empresariales deben participar activamente en la formación integral del estudiantado universitario, ofrecer un servicio educativo acorde a las necesidades, requerimientos y retos del contexto local, nacional e internacional, considerando el momento actual y el futuro, para el desarrollo de la sociedad con la labor del profesional graduado.

Desde la familia se deben practicar, según Tobón, los siete deberes esenciales del proceso de convivencia y crecimiento, estos son el liderazgo, el contacto afectivo, la autorreflexión, el diálogo, la autorrealización, el emprendimiento y la responsabilidad personal que implica la autogestión de un proyecto ético de vida propiamente dentro de este seno, el cual es muy importante dentro de la sociedad y por supuesto en la comunidad universitaria (2015).

En el contexto universitario, se hace necesaria la formación de profesionales investigadores, idóneos para comprender científicamente y transformar la problemática social, saliendo del claustro universitario para vin- cularse con los gobiernos de desarrollo local, con las organizaciones de la sociedad civil, con las cámaras de la producción, con la comunidad, con la familia; en fin, con todo el pueblo, de donde sale y al que se debe.

El Modelo de Salud Pública en Cuba ha tenido un desarrollo de connotación mundial como pionera en aspectos de la medicina, la pedagogía, la investigación y la docencia de pregrado y postgrado. En el ámbito de la Salud Pública, creando un sistema de salud único bajo las directrices del Ministerio y con la voluntad política que se requiere, como necesidad, para desplegar los niveles de atención y los principios que rigen este sistema.

La superación continua de los profesionales se convierte, por tanto, en un problema social, pues se considera que la salud es un derecho que tiene el pueblo y los cambios positivos desde el contexto universitario provocan que se transite por el camino correcto, a medida que surjan innovaciones que conduzcan a la integralidad del proceso educativo en las universidades de la sociedad actual. Muchos países han seguido este estilo de formación de profesionales, igual al de Cuba, como son países europeos, como España, que sigue un esquema de formación de medicina familiar, así también países pertenecientes a la Alternativa Bolivariana para las Américas (ALBA).

De igual manera, se manifiesta esta situación en otras universidades, como es el caso de la Universidad Nacional de Chimborazo, en Ecuador, donde se aúnan esfuerzos por alcanzar una categoría de universidad de excelencia, lo que incide en la necesidad de formar un profesional integral, preparado para enfrentar y resolver los problemas sociales de la comunidad universitaria y la población.

\section{Salud comunitaria. Conceptualización.}

La Organización Mundial de la Salud en las conferencias de la Carta de Ottawa para la Promoción de la Salud en el 1986, define a la salud como "el estado de completo bienestar físico, mental y social, y no solo la ausencia de enfermedad" (OMS 1986). Esta de- 
finición incorpora el factor social como elemento que también conforma el bienestar de las personas, además de los factores físicos y mentales, tradicionalmente considerados. El factor social es además un elemento determinante de la calidad de vida y el bienestar de las personas que pasa a depender, no solo del mundo de la sanidad, sino también del mundo social. En esta época la promoción de salud, prevención de enfermedades, curación y rehabilitación, se convierten en las actividades más idóneas para proporcionar salud a los ciudadanos.

A partir de esta nueva concepción se fomentan las actividades de educación para la salud y atención de las personas. La Carta de Ottawa, en 1974, expone como una nueva perspectiva para la salud los siguientes determinantes: medio ambiente, estilo de vida, biología humana y sistema de asistencia sanitaria.

\section{Comunidad.}

Etimológicamente el término comunidad proviene del latín comunitas y expresa la calidad de común, de lo que (no siendo privativo de uno solo) pertenece o se extiende a varios. Así pues, dependiendo de lo que se considere común o compartido, podrán identificarse diversos tipos de comunidades (Ander 2007).

El concepto de comunidad más utilizado en las Ciencias Sociales, plantea que es la agrupación o conjunto de personas vinculadas entre sí, que habitan en espacios geográficos delimitados y delimitables (política-administrativa, histórica-cultural) que pueden ser por barrios, repartos, municipios, regiones, pueblos, provincias y hábitat que son fácilmente reconocibles y que se perciben como una unidad social, que operan en redes relativamente estables dentro de la comunidad y su contexto. Parafraseando al doctor Canals, quien dijera que los miembros tienen coincidencia de pertinencia, religión o identificación con algún símbolo local e interacciones entre sí, más intensamente que en otro contexto (Canals 1991).

Es decir, es un conjunto, asociación o agru- pación de individuos, que tienen conciencia de pertenencia y que operan en redes de comunicación, intereses y apoyo mutuo, para satisfacer necesidades sentidas, desempeñar funciones establecidas y una estrecha vinculación con el entorno, el cual protegen y en el que se desarrollan.

\section{Acercamiento a la conceptualización de competencias.}

La competencia puede ser vista como capacidad, integración, conjunto, repertorio, configuración psicológica, combinación de conocimientos, destrezas, habilidades, actitudes, procedimientos, atributos, valores, hasta aspectos intelectuales, prácticos, éticos, actitudinales, afectivos, volitivos, estéticos y sociales para un desempeño eficiente (Glazman 2005). Las competencias cada día tienen mayor posicionamiento en la educación. Han pasado de ser un elemento secundario y fuertemente criticado, a convertirse en un concepto clave de la formación en todos los niveles. También muchas universidades la están abordando en el diseño de los planes de estudio.

\section{Competencias en el enfoque socio for- mativo.}

Se entiende como enfoque socio formativo el marco de reflexión-acción educativo que genera las condiciones pedagógicas esenciales para la formación de íntegros, integrales y competentes a partir de la articulación de la educación con los procesos sociales comunitarios. Tiene como propósito el establecimiento de recursos y espacios para promover la formación humana integral y con competencia para actuar con idoneidad en los diferentes contextos, considerando las dinámicas sociales y económicas.

Las competencias que tienen un enfoque socio formativo asumen el reto de la ordenanza ética en todos los espacios instructivos, debido a que la ética no se considera como una competencia, sino como la esencia estructurante de todas ellas. Son actuaciones integrales de las personas frente a actividades 
y problemas del contexto con mejoramiento continuo, ética, e idoneidad; en tanto articulan los saberes (saber ser, saber convivir, saber conocer y saber hacer) con el manejo de las situaciones externas, asumiendo los cambios y la incertidumbre con autonomía y creatividad.

En el enfoque socio formativo el conocimiento sobre el objeto se analiza en relación con el sujeto y se realiza una observación sobre la observación, es decir, se analizan los efectos de las propias actitudes y modelos mentales en la elaboración del conocimiento, el diseño de la metodología y su aplicación.

La socio-formación implica que la sociedad en su conjunto posibilita espacios, recursos, estrategias, apoyos, finalidades, normas, demandas, expectativas y valores, para medir la formación de sus miembros; con el fin de mantenerse y reconstruirse continuamente, afrontando los cambios dentro del marco de determinados contextos (Tobón 2015).

\section{Competencias comunicacionales. Salud Comunitaria.}

En estas menciones se hace hincapié respecto a la promoción de salud y prevención de enfermedades en el nivel primario en la formación de los gestores, para que adquieran competencias comunicativas pertinentes durante su formación académica regular. Es de interés marcado la integralidad en la formación del profesional de la salud, como gestor comunicacional de salud comunitaria, que no es más que un profesional preparado para hacer promoción de salud, con calidad y calidez educativa (dar calidez en educación significa, además, involucrar e incorporar sensaciones, sentimientos, emociones, afectos y desafectos, filias, fobias, formas de percepción), tanto en el plano pedagógico, como salubrista.

De análisis del planteamiento anterior, se deduce que la promoción de salud contribuye a la educación para la salud y la prevención de enfermedades en la comunidad. De forma tal que este gestor comunicacional, trate las cuestiones relacionadas con factores de riesgo, determinantes de salud y enfermedades que existen en la comunidad, para mejorar la calidad de vida.

Por otro lado, cada vez son mayores las expectativas de la población respecto a la necesidad de ser objeto de medidas preventivas y de promoción de la salud y por ello, es predecible que, progresivamente, vayan reclamando a los profesionales de Atención Primaria su realización.

Todo ello hay que ponerlo en relación con un grado de aceptabilidad cada vez mayor respecto a este grupo de actividades, sobre todo hacia aquellas que no supongan intervenciones instrumentales molestas y/o complejas. Tampoco podemos olvidar que el hecho de que estas medidas no supongan un costo adicional directo para el usuario, por estar comprendidas dentro de las habituales del sistema público de salud, favorece su implantación y cumplimiento (Zurro 2015).

En tal sentido, y como parte de la denominada Misión Barrio Adentro, en Venezuela, se desarrolló una experiencia, a partir de conformar grupos de promotores comunitarios, que posteriormente integraron las filas de estudiantes de Medicina Integral Comunitaria, lo que permitió acercar a los futuros médicos a la comunidad, evidenciando la necesidad de que los profesionales de la salud, requieren desarrollar habilidades comunicativas y pedagógicas, para incidir notablemente, en la educación para la salud y mejorar la calidad de vida de la población.

Con la experiencia de Venezuela, en Ecuador, la misión médica cubana integró en el programa de especialización de médicos familiares y comunitarios, módulos de promoción para la salud, donde se implementaron metodologías y técnicas para fortalecer las habilidades comunicativas.

Al respecto, se debe señalar que la Escuela Superior Politécnica de Chimborazo, contaba con el programa de Licenciatura en Educación para la Salud, sin embargo, no se ha logrado reconocer formalmente a este profesional, dentro de los Equipos Básicos de Salud (EBS), lo que constituye otra evidencia que enfatiza la importancia de ejecutar un proyecto de formación integral de los profesionales de la salud.

Tomando en cuenta el análisis anterior y en apego a los parámetros de multiplicidad de factor, establecidos por Tobón en el 2015, el autor de este artículo concibe que el proceso 
de formación de los profesionales de la salud, debe incluir la formación de los mismos como gestores comunicacionales de salud comunitaria, lo cual constituye un sistema complejo, que debe considerar la unidad entre varios elementos, tal y como se evidencia en la Tabla 1, a ser considerados por las autoridades encargadas de la formación.

Tabla 1: Proceso de formación de gestores comunicacionales de salud comunitaria.

\begin{tabular}{|c|c|}
\hline Finalidad & Formar gestores comunicacionales de salud comunitaria, con integralidad. \\
\hline Entorno & Comunitario \\
\hline $\begin{array}{l}\text { Tipos de } \\
\text { Educación }\end{array}$ & Superior y Continua \\
\hline $\begin{array}{l}\text { Modelos } \\
\text { Pedagógicos }\end{array}$ & Socio formativo por competencias \\
\hline $\begin{array}{l}\text { Relación con } \\
\text { el entorno }\end{array}$ & $\begin{array}{l}\text { Abierto en cuanto a las necesidades y retos sociales de cada comunidad y el } \\
\text { entorno. } \\
\text { Cerrado en cuanto a las metodologías y filosofias propias de las universidades } \\
\text { que intervienen. }\end{array}$ \\
\hline Resultados & $\begin{array}{l}\text { Se esperan alcanzar: } \\
\text { Gestores capaces de mejorar su calidad de vida y la de la comunidad en la cual } \\
\text { podrán desempeñarse. } \\
\text { Comunidades saludables con indicadores de salud adecuados. } \\
\text { Visibilidad de los factores de riesgo para evitar enfermedades. } \\
\text { Liderazgo del gestor en su comunidad y entorno. } \\
\text { Acción profesional ética, que evidencie la formación integral. }\end{array}$ \\
\hline Intereses & $\begin{array}{l}\text { Político: cumplimiento de los objetivos y/o propósitos de Salud Pública. } \\
\text { Filosófico: mantener la adecuada calidad, estilo y modo de vida en la } \\
\text { comunidad, individuo y familia con el entorno. } \\
\text { Económico: reducir los costos para la atención secundaria y terciaria. } \\
\text { Social: fortalecer las condiciones de salubridad pública y comunitaria, } \\
\text { teniendo como lider formal al gestor comunicacional, en coordinación con los } \\
\text { líderes no formales. } \\
\text { Comunitario: fortalecerse desde el punto de vista de salud, como núcleo social } \\
\text { capaz de generar sus propias transformaciones y mejora la calidad de vida de } \\
\text { la población. }\end{array}$ \\
\hline $\begin{array}{l}\text { Relación } \\
\text { entre los } \\
\text { componentes }\end{array}$ & $\begin{array}{l}\text { Hay una interacción continua entre los componentes, los cuales son: } \\
\text { Individuos: se forman como gestores comunicacionales de salud comunitaria } \\
\text { para cambiar su estilo de vida y el de sus personas atendidas. } \\
\text { Familia: mejora el modo de vida. } \\
\text { Comunidad: mejora la calidad de vida. } \\
\text { Universidades: aporta con la formación de los gestores y se vincula a la } \\
\text { sociedad. }\end{array}$ \\
\hline
\end{tabular}

Fuente: Elaboración según los datos recopilados en la observación y la bibliografia, a partir de la obra de Tobón (2015). 
Como argumento adicional a los datos expuestos en la tabla 1, es preciso mencionar que cada época, ciencia y proceso social han dado y dan una respuesta diferente a la formación humana, la cual "es un proceso complejo que representa un desafío para las concepciones epistemológicas tradicionales" (Lizarra 1998:156).

Lo que significa que no es posible pensar la formación humana en el marco de propuestas epistemológicas, unidimensionales, simplistas y unívocas, por lo cual el pensamiento complejo constituye una perspectiva de relevancia para la reconceptualización.

Esta concepción aplicada a la formación de los profesionales de la salud, constituirá insumo fecundo para la divulgación, a través de artículos científicos, como resultado de las investigaciones que se realicen en la comunidad y se logrará la formación teórica-práctica del futuro profesional de la salud, como gestor comunicacional de salud comunitaria.

Con la formación de este profesional integral, con equidad cognoscitiva, tanto desde el punto de vista salubrista como pedagógico, se puede fomentar la mejora en la visión de riesgo de la población para no enfermar, es decir la prevención de enfermedades, conociendo los factores de riesgo que propician las enfermedades comunes y las crónicas, además de facilitar el cambio del estilo, modo y calidad de vida.

\section{CONCLUSIONES}

Estudios realizados en diversos contextos, exponen la importancia de la formación por competencias, sin embargo, con la excepción de algunos países que integran el ALBA, la formación de profesionales de la salud en la actualidad, adolece en general, de un sistema integrado de conocimientos y competencias comunicacionales tanto de las Ciencias de la Salud, como de las Ciencias Pedagógica, para formarlos como gestores comunicacionales de salud comunitaria, para lo cual es necesario que adquieran y/o desarrollen competencias comunicativas que les permitan interrelacionarse en sus contextos laborales con efectividad, de manera que su accionar profesional logre el impacto en la comunidad para mejorar la calidad de vida de la población.

Esta formación incidirá favorablemente en la solución de los problemas existentes en las comunidades, pues la ciencia no solo es una actividad teórica, es una actividad social institucionalizada, portadora de valores y cultura, que transita de actividades comunicativas a actividades desarrolladoras, o sea, de actividades cognitivas y axiológicas a actividades prácticas en la sociedad, aplicando principios conceptuales, conocimientos salubristas y estrategias pedagógicas. 


\section{REFERENCIAS BIBLIOGRÁFICAS}

Adler, N. E., Boyce, T., Chesney, M. A., Cohen, S., Folkman, S., Kahn, R. L. \& Syme, S. L. (1994). Socioeconomic status and health: The challenge of the gradient. American Psychologist, 49(1), 15-24.

Alarcón, R. (2014). Universidad socialmente responsable. Conferencia inaugural 9no. Congreso Internacional de Educación Superior. La Habana, Cuba: Ministerio de Educación Superior.

Ander, E. (2007). Acción Municipal, desarrollo local y trabajo comunitario. Caracas. Venezuela: Imprenta UBV.

Atria R. \& Siles M. (2003). Capital social y reducción de la pobreza: en busca de un nuevo paradigma. Santiago de Chile: CEPAL; Universidad del Estado de Michigan.

Bracht, N. (1999) Health promotion at the community level: new advances. (Segunda ed.). Newbury Park: SAGE.

Canals, J. (1991). Comunidad y redes sociales: de la metáfora a los conceptos operativos. Revista de Servicios Sociales y Político Social, 23.

Glazman, C. (2005). Las caras de la evaluación educativa. México DF: Editorial PAIDEIA.

Horruitiner, P. (2005). La Universidad cubana: el modelo de formación. Cuba: Editorial Félix Varela.

Lalonde, M. (1974). A new perspective on the health of $\mathrm{Ca}$ nadians. Ottawa: Health and Welfare Canada.

Lizarra. (1998). Acuerdo de Lizarra. Navarra de Estella.

Organización Mundial de la Salud.(OMS) (1986). Carta de Otawa para la Promoción de la Salud. Conferencia Internacional auspiciada por la OMS y la Asociación Canadiense de Salud Pública. Toronto. Canadá.

Martínez, J. (2009). Gripe A. Pandemia Gripal . Madrid.

Tobón, S. (2015). Formación integral y competencias: pensamiento complejo, currículo, didáctica y evaluación. Bogotá.

Zurro, M. (2015). Actividades preventivas y promoción de la salud. Madrid, España.

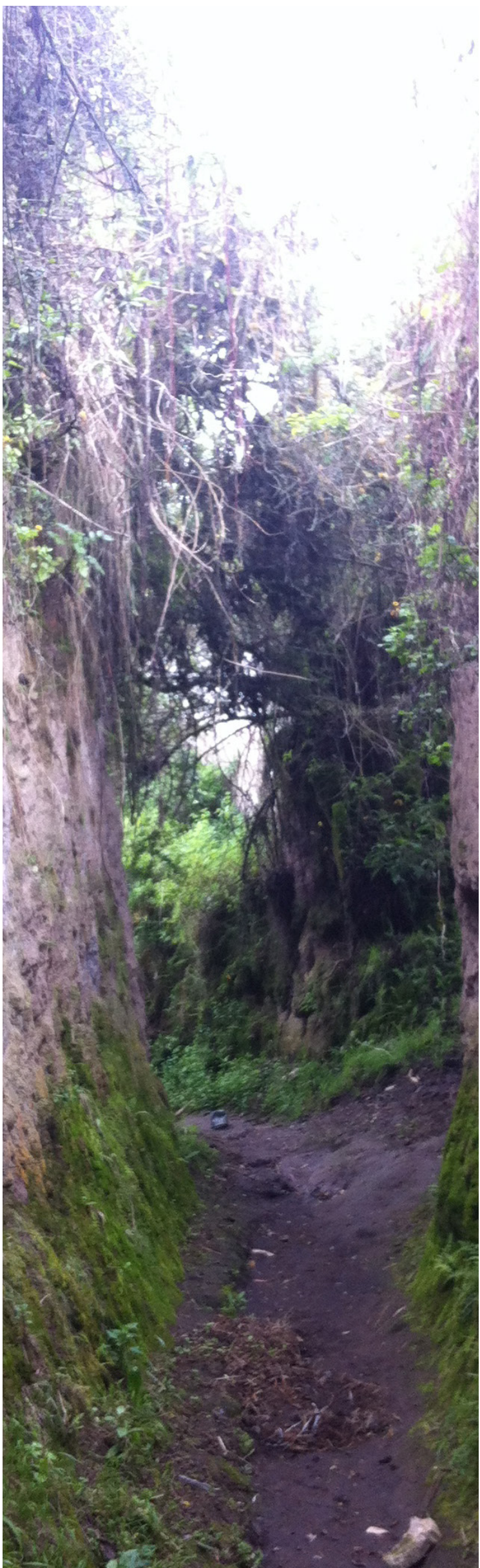

回 $>\frac{\text { 回回 }}{93}>$ 回

